\title{
Klimaforschung und Politikberatung - zwischen Bringeschuld und Postnormalität
}

\author{
Hans von Storch
}

Zusammenfassung: Die naturwissenschaftlichen und populären Wissensansprüche in Bezug auf Klima, Klimawandel und Klimawirkung unterscheiden sich erheblich; dies hat Folgen für die naturwissenschaftliche Beratung von Politik und Gesellschaft. Wie üblich in einer postnormalen Phase einer Wissenschaft sieht sich die Klimaforschung von verschiedenen Interessengruppen instrumentalisiert. Zusammen mit dem Gebot der Bringeschuld entsteht so eine Mischung aus Politik und Wissenschaft, wobei die spezifischen Aufgaben und Leistungen der Wissenschaft verschwimmen. Hier ergeben sich wichtige Aufgaben für eine sozialwissenschaftliche Begleitforschung, die es bisher nur vereinzelt gibt. Abschließend werden die Bemühungen des GKSS Forschungszentrums beschrieben, dennoch eine unabhängige wissenschaftlich basierte Beratungsleistung zu erbringen.

Schlüsselwörter: Klimaforschung · Wissen · Postnormalität · Bringeschuld · Klimapolitik · Klimaberatung

\section{Climate research and policy advice between Bringeschuld and postnormality}

\begin{abstract}
Scientific and popular knowledge claims about climate, climate change and climate impact differ significantly from each other. These different views have implications for the scientific advising of society and policy making. Climate science is being used by various social groups as support for achieving their interests - as is common for a post-normal phase. The concept of "Bringeschuld" adds a momentum of urgency to many issues, so that the public is flooded by alarming views. In this situation, the analytical skills of social sciences are urgently needed to describe and understand the dynamics of knowledge generation and transformation; a few examples of successful studies of that sort are discussed. Finally, the efforts at the research laboratory GKSS is trying to provide an independent, science-based policy advice are sketched.
\end{abstract}

Keywords: Climate research $\cdot$ Climate change $\cdot$ Policy advice $\cdot$ Climate policy $\cdot$ Postnormality Bringeschuld

(C) VS-Verlag 2009

Prof. Dr. Dr. he H. von Storch $(\bowtie)$

Geomatikum, Meteorologisches Institut der Universität Hamburg, Bundesstraße 55,

20146 Hamburg, Deutschland

E-Mail: hvonstorch@web.de

http://coast.gkss.de/staff/storch 


\section{Wissen über Klimawandel}

Die Wissenschaft hat festgestellt, dass vom Menschen ausgehende Prozesse das Klima beeinflussen - dass der Mensch das globale Klima verändert. Das Klima, das ist die Statistik des Wetters. Die Häufigkeitsverteilungen der Temperatur verschieben sich derzeit und in der absehbaren Zukunft fortgesetzt an fast allen Orten hin zu größerer Wärme; der Meeresspiegel steigt; die Regenmengen verändern sich. Auch einige extreme Wetterereignisse, wie etwa Starkniederschläge im Westwindgürtel der mittleren Breiten, werden sich in Häufigkeit und Intensität in Zukunft verändern. Diese Veränderungen sind verursacht vor allem durch die Freisetzung von Treibhausgasen, also insbesondere Kohlendioxid und Methan.

Dies ist das wissenschaftliche Konstrukt des menschgemachten Klimawandels. Es findet breite Unterstützung in den einschlägigen wissenschaftlichen Kreisen und wird insbesondere durch die kollektive Anstrengung des UNO-Klimarats „IPCC“"1 formuliert.

Was wissen wir noch über Klima und Klimawandel? Dass das Klima sich wirklich wegen des Menschen ändert, auch z.B. durch Entwaldung. Dass das Wetter weniger zuverlässig ist als früher, die Jahreszeiten unregelmäßiger, die Stürme gewaltiger. Die Wetterextreme nehmen katastrophale, vorher nie gewesene Formen an.

Fragt man nach der Ursache, so stößt man auf „menschliche Gier“ und „Dummheit“" als Antwort. Das sei der Mechanismus der Gerechtigkeit, der Rache der Natur, die zurückschlägt. Manchmal ist dann auch von Gott selbst die Rede. So berichtet Frances Welch unter der Überschrift „Me and My God“ in Sunday Telegraph (10.9.95) über den früheren IPCC Vorsitzenden Sir John Houghton: "An expert on global warming and Chairman of the Royal Commission of Environmental Pollution, Houghton warns that God may induce man to mend his ways with a disaster. 'God tries to coax and woo, but he also uses disasters.' Und: 'If we want a good environmental policy in the future we'll have to have a disaster.” Auf dem Umschlag eines einschlägigen Buchs „Our drowning world“ aus den 1990er Jahren heißt es ,,[...] we shall be engulfed by the consequences of our greed and stupidity. Nearly two thirds of our world could disappear under polar ice cap water [...] For this will be the inevitable outcome of industrialization, urbanization, overpopulation and the accompanying pollution“" (Milne 1989, Buchumschlag).

Bei den Antworten auf die Frage nach der Wirkung des Klimawandels spielt latent eine Rolle immer wieder der klimatische Determinismus (z. B. Stehr u. von Storch 1999b, S. 137-185), von dem weiterhin Elemente in unserer westlichen Kultur wirksam sind. Eines dieser Elemente ist das Wissen, dass der Mensch im Gleichgewicht mit seinem ihm angemessenen Klima leben muss. Ändert sich dieses Klima, dann ist die Zivilisation gefährdet; ganze Kulturen gingen bei solchen Ereignissen unter, etwa Indianerkulturen in Nordamerika oder Wikinger-Siedlungen in Grönland. Es wundert daher nicht, dass es im deutschen Sprachgebrauch oft „Klimakatastrophe“ heißt und nicht „Klimawandel“.

Dies ist das kulturelle Konstrukt des Klimawandels.

1 Intergovernmental panel on climate change, http://www.ipcc.ch. 
Das wissenschaftliche und das kulturelle Konstrukt ${ }^{2}$ sind Konkurrenten in der Deutung einer komplexen Umwelt; zwei „Akteure“ auf dem Markt des Wissens. Wenn die beiden Formen zusammengebracht werden, mag die Wirkmächtigkeit des so entstehenden modernisierten Konstrukts wachsen; seine wissenschaftliche Basis aber wird schmaler. Die öffentliche Akzeptanz steigt, seine Robustheit gegenüber wissenschaftlich nachprüfbaren Fakten sinkt.

Natürlich wird die naturwissenschaftliche Praxis (und damit ihre Theoriebildung) ohnehin durch das kulturelle Konstrukt beeinflusst, weil die Naturwissenschaftler ja eben gar nicht frei von ihrer Kultur sein können. Ihre Kultur konditioniert sie in ihrer Sichtweise, leitet sie in ihren Fragestellungen, in ihrer Bereitschaft, Antworten als argumentativ ausreichend anzusehen.

\section{Die Bringeschuld und die Arena der Aufmerksamkeit}

Irgendwann in den 1960ern oder 1970ern wurde in Deutschland der Begriff der Bringeschuld geschaffen - dabei handelt es sich um die ethische Verpflichtung gerade auch der Naturwissenschaftler, die Gesellschaft zu informieren über existierende, entstehende und mögliche zukünftige Gefahren. In der Vergangenheit hatte die Naturwissenschaft allzu oft die Augen geschlossen vor solchen Gefahren und sich so zum willigen Handlanger wissenschaftlich-technologischer Entwicklungen sowie politischer und gesellschaftlicher Interessen wie Eugenetik und Atomkraft gemacht. Belohnt durch Einnahmen, Anerkennung und die Befriedigung bisweilen perverser Neugier war tatenlos und verantwortungslos zugesehen worden. Damit sollte Schluss sein. Die Wissenschaftler sollten ihr Tun in einem gesellschaftlichen Rahmen sehen; die Öffentlichkeit von sich aus informieren, unaufgefordert - damit diese dann in demokratischer Praxis entscheiden könne über Sinn und Unsinn.

Was wissen die Wissenschaftler, die ja Experten in ihrem Gebiet sind, sonst aber Laien wie jeder andere auch, über die Gefahren? Oft ist es so, dass die wahrgenommenen Gefahren außerhalb des Expertenbereichs des jeweiligen Wissenschaftlers liegen, d.h. auch der Experte operiert mit kulturell konstruiertem Wissen, nicht aber wie die betrachtende Öffentlichkeit glaubt, mit wissenschaftlich konstruiertem Wissen. Es ist also oft nicht das beste Wissen, was zum Zuge kommt, sondern vielmehr beanspruchtes Wissen. Als Wissenschaft verkleidete Deutungs- und Machtansprüche.

Es gibt nicht wenig echte oder wahrgenommene Gefahren; es gibt vielmehr viele derartiger Gefahren. Sie treten miteinander in Konkurrenz um die öffentliche Aufmerksamkeit. Die Öffentlichkeit aber kann nur eine begrenzte Anzahl von Themen dauerhaft ,,verarbeiten"; wie viele ist unklar, aber gewiss wäre mehr als zehn eher unwahrscheinlich. Einige sind dabei vorgegeben, etwa die Fußballbundesliga. Wie werden die sichtbaren Themen ausgewählt? Man würde hoffen, das entscheidende Kriterium wäre die Dringlichkeit, aber das ist ganz sicher nicht der Fall. Vielleicht ist es der Unterhaltungswert, auch der

2 Tatsächlich sind es natürlich viele verschiedene Konstrukte, aber der Einfachheit halber seien hier diese beiden idealisierten Formen gegeneinander gestellt. 
Angstmachwert, die Herausforderung oder auch die Sicherung der Deutungsordnung, dass mit dem kulturellen Konstrukt alles in Ordnung ist.

Aber man kann natürlich auch versuchen, das eigene Thema, für das man Experte ist, in die Arena der öffentlichen Aufmerksamkeit zu katapultieren. Die dazu erforderlichen Attribute müssen dann hinzugefügt werden, etwa durch Übertreibung, durch nichtausgesprochene Assoziationen, durch Ausnutzung des kulturellen Konstrukts, also dessen, was die Öffentlichkeit ohnehin als richtig erkennt. Das „Waldsterben“ war ein nach diesem Muster konstruiertes Thema.

Ob die Erfüllung der Bringeschuld für das Individuum nützlich oder schädlich ist, hängt vom gesellschaftlichen Kontext ab. Als jedermann vom wissenschaftlich-technischen Fortschritt begeistert war, als in den „Micky Maus“-Heften in der Reihe „Unser Freund, das Atom" dem jugendlichen Publikum eine goldene Zukunft mit der allgegenwärtig nutzbaren Kernenergie beschrieben wurde, da wurde der Hinweis auf die Kehrseiten diesen Fortschritts nicht beachtet. Heute aber, mit einer skeptischen Haltung gegenüber dem wissenschaftlich-technischen Fortschritt, insbesondere wenn er sich im unmittelbaren Umfeld dokumentieren sollte in Form von Masten, Geräuschen oder Gerüchen, wird eine wissenschaftlich vorgetragene Gefahreneinschätzung gesellschaftlich honoriert, auch gerade weil diese ja das Vorwissen bestätigt und damit a priori ohnehin als richtig anerkannt wird. Dieses Honorieren kann mannigfache Formen annehmen: Karriere, öffentliche Aufmerksamkeit und Anerkennung, bessere Arbeitsbedingungen, persönliche Befriedigung aus dem Glauben, aus der Welt einen besseren Ort gemacht zu haben.

Die Bringeschuld zu befriedigen, ist heutzutage oft kein altruistischer Akt mehr, sondern eine zielführendes Element in einer Marktstrategie. Die Bringeschuld hat zu einem massenhaften Strom an beschworenen Gefahren in die Arena der öffentlichen Aufmerksamkeit geführt. Umweltwissenschaft, und nicht nur diese, ist ,postnormal“ geworden.

\section{Postnormale Wissenschaft und der Bedarf von Politik und Medien}

Der Begriff ,postnormal“" wurde von dem Silvio Funtovitz und Jerome Ravetz in den 1980er Jahren in die Analyse eingebracht (vgl. Funtowicz u. Ravetz 1985, S. 217-231). In einer Situation, wo Wissenschaft in ihren konkreten Aussagen unsicher bleiben muss, und in der die Aussagen der Wissenschaft von erheblicher praktischer Bedeutung für die Ausformulierung von Politik und Entscheidungen sind, wird diese Wissenschaft immer weniger von reiner „Neugier“ getrieben, die in idealistischer Verklärung als innerste Triebfeder von Wissenschaft dargestellt wird, sondern von der Nützlichkeit der möglichen Aussagen für eben die Formulierung von Entscheidungen und Politik. Nicht mehr die Wissenschaftlichkeit steht im Zentrum, die methodische Qualität, das Poppersche Falsifikationsdiktum oder auch der Flecksche Reparaturbetrieb überzogener Erklärungssysteme (vgl. Fleck 1980), sondern die Nützlichkeit. „Nichts ist so praktisch wie eine gute Theorie“, sagte Kurt Lewin (Lippitt 1968, S. 266-271) dazu und verwies damit auf die Fähigkeit, Entscheidungen zu ermöglichen, Handlungen zu leiten. Nicht die Richtigkeit oder die objektive Falsifizierbarkeit steht im Vordergrund, sondern die soziale Akzeptanz.

Naturwissenschaft in ihrer postnormalen Phase lebt also auch von ihren Ansprüchen, ihrer medialen Inszenierung, ihrer Konsistenz mit kulturellen Konstruktionen. Die Wis- 
sensansprüche werden nicht mehr nur von ausgewiesenen Wissenschaftlern erhoben, sondern auch von anderen selbsternannten Experten, die häufig genug auch speziellen Interessen verpflichtet sind, seien sie nun Exxon oder Greenpeace.

Klimaforschung ist derzeit postnormal. Die inhärenten Unsicherheiten sind enorm, da Projektionen für die Zukunft verlangt werden - also Beschreibungen von möglichen Zukünften, die nur mit Modellen dargestellt werden können, wo Bedingungen herrschen werden, die bislang nicht beobachtet wurden. Man weiß eben nicht genau, wie sich die Bewölkung verändern wird, wenn Temperaturen und Wasserdampfgehalt sich ändern, wer in Bezug auf den Massenhaushalt der Antarktis die Oberhand gewinnen wird - der vermehrte Niederschlag in der Höhe oder das Abschmelzen am Rande. Dieser Mangel an Wissen hat nichts mit Unfähigkeit der Wissenschaftler zu tun, sondern mit der dürftigen Faktenlage, mit den unvollständigen instrumentellen Daten, die für die Betrachtung von Veränderungen auf Zeitskalen von Jahrzehnten einen viel zu kurzen Zeitraum überspannen, mit den durchaus problematischen Proxydaten, die nicht nur Klimaschwankungen sondern auch alles mögliche andere darstellen. Sicher, es gibt Argumente, die auf die eine oder andere Antwort verweisen, und Plausibilitätsbetrachtungen lassen uns gewisse Entwicklungen als unwahrscheinlich oder gar unmöglich ausschließen. Es bleibt aber eine Restunsicherheit, die sich erst im Laufe der Jahre und Jahrzehnte deutlich vermindern wird.

In dieser Lage suchen sich die Vertreter gesellschaftlicher Interessen jene Wissensansprüche heraus, die ihre Positionen am besten stützen. Man denke an den Stern-Report (z. B. Pielke 2007 oder Yohe u. Tol 2008), der selektiv Abschätzungen zu Klimaschäden zusammenstellt, oder US-Senator Inhofe, der einen regelmäßigen e-mail-Dienst betreibt mit nur einer Aussage, nämlich, dass es einen signifikanten menschgemachten Klimawandel nicht gäbe und es sehr viel Wissenschaftler gäbe, die sich dieser Linie anschlössen. Aber nicht nur werden die geeignet erscheinenden Wissenselemente ausgewählt und in ein passendes Gesamtbild gestellt; auch eigene neue Wissensansprüche werden konstruiert, so dass am Ende eine wilde Ansammlung von manchmal beliebig erscheinenden Behauptungen entsteht, etwa dass es vermehrt Patienten mit Nierensteinen (vgl. Brikowski et al. 2008) geben werde als Folge der menschgemachten Erderwärmung. Der wissenschaftlich unhaltbare Film „The day after tomorrow“ (2004) wird von öffentlich sichtbaren Wissenschaftlern als bewusstseinsfördernd gelobt; politische und wissenschaftliche Leistungen werden durch die gemeinsame Verleihung des Friedensnobelpreises an Al Gore und das IPCC vermischt; als Professoren verkleidete Politiker erklären der Öffentlichkeit notwendige Maßnahmen als Reaktion auf den Klimawandel. Neben diesen alarmistischen Tendenzen gibt es auch das skeptische Pendant, das sich in Produkten wie „State of fear“ (2004) des für seine frühen Bücher gefeierten Michael Crichton oder dem Film „The great global warming swindle“ (2007) darstellt. All dies ist typisch für eine postnormale Wissenschaft.

Dem eigenen Anspruch der Naturwissenschaft kann dieser Zustand nicht genügen. Im Tagesgeschäft ergeben sich viele Möglichkeiten sowohl für den Einzelnen wie für mächtige Wissenschaftsorganisationen, die Aufmerksamkeit der Öffentlichkeit auf sich zu ziehen. Aber es bleibt ein gewisses Nagen des Gewissens, dass diese Praxis eben nicht das sein kann, was wir ungenau mit ,gute Naturwissenschaft“" umschreiben, wo es das Argument, die kritische Nachfrage, der kluge Test, die unkonventionelle Idee jenseits 
des geltenden Paradigmas ist, die den Fortschritt bewirken und nicht die Nützlichkeit zur Durchsetzung einer als richtig wahrgenommenen oder beschriebenen Politik. Selbst in Science und Nature erscheint viel Halbgares, das die Phantasie und manchmal die Ängste des gebildeten Publikums anregt - und sich nach einigen Jahren dann oft eben doch als revisionsbedürftig erweist. Aber diese Revision ist nichts anderes als der Mechanismus, der die Wissenschaft aus dem Strudel der Postnormalität herausholt. Wenn die Karawane der öffentlichen Aufmerksamkeit sich anderen Themen zuwendet, dann greift die normale Naturwissenschaft wieder, und die Kompromisse an die erforderliche Nützlichkeit, den Zeitgeist und die politische Korrektheit können revidiert werden. Im kleineren Maßstab sehen wir das schon jetzt in der Klimaforschung, etwa im Falle der als „Hockeystick““3 beschriebenen voreiligen Schließung der Frage nach historischen Temperaturschwankungen oder der von der Versicherungswirtschaft geförderten Wahrnehmung eines verschärften Sturmrisikos.

\section{Eine Rolle für Sozial- und Kulturwissenschaften}

Für uns als beteiligte Naturwissenschaftler stellt sich die Frage, wie wir hier und heute mit dieser postnormalen Situation umgehen, denn beide Forderungen - gute Naturwissenschaft und gute Beratung von Öffentlichkeit - akzeptieren wir als berechtigt. Die Lösung kann eigentlich nur darin bestehen, dass wir das tun, was wir zumindest im Prinzip am besten können, nämlich die Situation wissenschaftlich zu analysieren. Aber wir Naturwissenschaftler können das nur im beschränkten Maße. Wir ahnen schon, dass der Prozess der Wissenschaft ein sozialer Prozess ist, dass wir zumindest beim Fragen und beim Akzeptieren von Erklärungen nicht immer sehr objektiv sind, dass wir durch unsere Kultur konditioniert sind. Dass das Aufrücken von Einzelnen in wichtige Positionen oft weniger mit Wissenschaft sondern mehr mit sozio-politischer Akzeptanz zu tun hat.

Um der Analyse Tiefe und Substanz zu geben, brauchen wir also die Kompetenzen der Sozial- und Kulturwissenschaften. Aber bisher stehen diese Wissenschaften weitgehend abseits. Gelegentliche Hinweise, wonach alles sozial konstruiert und relativ sei, demonstrieren nur die fatale Weigerung, ins Konkrete zu gehen, was aber für eine wirkliche Synergie notwendig wäre. Ärgerlich ist, dass Kollegen aus diesen Bereichen offenbar das Auseinanderfallen von wissenschaftlichem und kulturellen Konstrukt nicht bemerken, sondern sich mit Wissensansprüchen begnügen, die der Tagespresse und dem Internet entnommen sind.

3 Als Hockeystick wurde eine Darstellung der Entwicklung der Temperatur im vergangenen Jahrtausend bezeichnet - mit einem glatten langen Schlägergriff, der eine langsame Abkühlung von 1000 bis ca. 1850 beschrieb und dann einen steilen Anstieg, der seitdem für den eigentlichen Schläger steht. Die Darstellung ging auf den Wissenschaftler Michael Mann zurück, der ihn in 1999 in Geophysical Research Letters veröffentlichte. Die Methodik, die hinter dieser Darstellung stand, erwies sich später als falsch (vgl. auch http://blogs.nature.com/climatefeedback/2007/05/the_decay_of_the_hockey_stick.html). 
Aber selbst wenn sich die überwiegende Mehrheit der Sozial- und Kulturwissenschaftler einem transdisziplinären ${ }^{4}$ Zugang zum Thema des menschgemachten Klimawandels noch verschließt, so gibt es doch hervorragende Beispiele, wo die erforderliche sozialwissenschaftliche Begleitforschung gelingt. Beispiele sind die Arbeiten des Medienwissenschaftlers Peter Weingart aus Bielefeld, des Politikwissenschaftlers Roger Pielke Jr. aus Boulder, sowie des Wissenssoziologen Nico Stehr aus Friedrichshafen (z. B. Stehr u. von Storch 1999b; 2008). Andere ermutigende Signale kommen aus dem Hamburger Exzellenzzentrum CLISAP, ${ }^{5}$ in dem eine Mehrheit von Naturwissenschaftlern mit einigen Sozialwissenschaftler zusammen arbeitet.

\subsection{Der ehrliche Wissensmakler}

In seinem Buch The Honest Broker hat Roger Pielke jr. (2007) eine Typologie von Wissenschaftlern aufgebaut und beschrieben, wie Politik und Wissenschaft zum Ersatzkriegsschauplatz degradiert werden, um Probleme zu lösen, die die Politik selbst nicht lösen kann - und die Wissenschaft ebenso wenig.

Pielke unterscheidet fünf Arten von Wissenschaftlern, die auf verschiedene Weise und in unterschiedlichem Maße in eine Kommunikation mit der Öffentlichkeit eintreten. Der „reine Wissenschaftler“ ist im Wesentlichen von Neugier getrieben und hat kaum Interesse, neue Erkenntnisse in einen gesellschaftlichen Kontext gestellt zu sehen. Der „wissenschaftliche Schlichter“ ermöglicht das richtige Verständnis unstrittiger wissenschaftlicher Fakten. Beide Typen passen gut zu einer „,normalen“ Wissenschaft, die Fragen mit großer Sicherheit beantworten kann, und bei gelegentlichen gesellschaftlichen Umsetzungen sind die entsprechenden praktischen Folgerungen in der Regel auch nicht kontrovers.

Aber wie vorhin ausgeführt, ist die derzeitige Klimaforschung nicht „,normal“, sondern „postnormal“. Daher sieht man oft den „wissenschaftlichen Anwalt“, der seine wissenschaftliche Kompetenz nicht zur unvoreingenommenen Fortschreibung des Wissens einsetzt, sondern zur Förderung einer wertorientierten, das heißt auch politischen Agenda. Dies bedeutet, dass Folgen wissenschaftlicher Einsicht verengt werden auf wenige, oder gar nur eine, wertkonsistente „Lösung“. Gerade die letzten Jahrzehnte haben viele Wissenschaftler dieses Typs hervorgebracht, die für wirtschaftliche oder (gesellschafts-)politische Interessen arbeiten und sprechen.

Der vierte Wissenschaftlertypus, den Pielke als Ideal ansieht, hat dem Buch seinen Namen gegeben: „Der ehrliche Makler“. Er zeichnet sich dadurch aus, dass er, anders als der ,wissenschaftliche Anwalt“, die Bandbreite der Folgerungen aus seinen Erkenntnissen verbreitert anstatt sie einzuengen. Dadurch ermöglicht er dem politischen Prozess, jene „Lösung“ auszuwählen, die gesellschaftlich gewollt ist (und nicht jene, die vom wissenschaftlichen Anwalt favorisiert und gefördert wird). Der fünfte Typ ist der ,verdeckte Anwalt“, der seinem Wirken nach ein ,wissenschaftlicher Anwalt“" ist, sich aber

4 Im Sinne von Zusammenarbeit von Naturwissenschaften auf der einen und von Sozial- und Kulturwissenschaften auf der anderen Seite.

5 Exzellenzclusters der Universität Hamburg „Integrated Climate System Analysis and Prediction (CliSAP)“, siehe http://www.clisap.de. 
als Schlichter oder ehrlicher Makler ausgibt. Der Sache nach tut er mit seinem Etikettenschwindel weder der Wissenschaft noch der Gesellschaft einen Gefallen.

Pielke empfiehlt der Wissenschaft, den Weg des ,ehrlichen Maklers“ zu gehen, der die Komplexität der Probleme darlegt und dazu beiträgt, die Implikationen von möglichen Entscheidungen abzuwägen. Dadurch versetzt er die Gesellschaft in die Lage, Lösungen für gesellschaftliche Kontroversen auch in Gegenwart unsicheren Wissens um Zusammenhänge und verschiedener Reaktionsoptionen rational so zu wählen, dass die Normen der Gesellschaft optimal bedient werden - etwa um mit der Perspektive des selbst verursachten Klimawandels umzugehen.

Die andere Frage ist die des Ersatzkampfplatzes. Wir erleben immer wieder Situationen, wo die Politik daran scheitert, zu Entscheidungen zu kommen, die in signifikant großen oder einflussreichen Gruppen als negativ wahrgenommen werden. In diesem Falle geschieht es, dass ein Sachzwang aufgebaut wird, wonach die Politik gemäß wissenschaftlicher Analyse nur eine Entscheidung treffen kann. Die Politik stellt sich dann als der Wissenschaft nachgeordnet dar. Gerade bei der Klimapolitik ist dies der Fall, wo das von Wissenschaftlern formulierte Zwei Grad-Ziel zur Vermeidung der Klimakatastrophe als ultima ratio dargestellt wird, der sich die Politik einfach beugen muss. Gemäß der Lewinschen Regel, wonach nichts so praktisch ist wie eine gute Theorie, ist diese Darstellung in der Tat politisch überaus nützlich, weil sie handlungsleitend ist. Weitere Diskussionen sind nicht erforderlich, die Ziele der Klimapolitik werden durch Energiepolitik erreicht. Dass Problem ist bloß, dass die Auseinandersetzung von der sichtbaren politischen Bühne in die öffentlich weniger sichtbare wissenschaftliche Diskussion verlagert worden ist. Dort ergibt sich für die zu ziehenden Folgerungen ebenso wenig ein Konsensus wie in der Politik, und der sich ergebende argumentative Kampf unter den Wissenschaftlern verkommt zu einer politischen Auseinandersetzung, die nach den Regeln der Politik geführt und schlussendlich von einer Partei ,gewonnen“ wird.

Der Politik nützt dieser Vorgang, kommt sie doch einfacher zu Entscheidungen, aber die Wissenschaft wird beschädigt, da sie politisiert wird. Dies ist keine nachhaltige Nutzung der Ressource „Wissenschaft“, deren soziale Dienstleistung, Deutung von komplexen Sachverhalten zu geben, in der öffentlichen Wahrnehmung dann kaum noch von der politischen Information von Interessenverbänden unterscheidbar wird.

Normativ leitet Pielke zwei Forderungen ab, nämlich, dass der verantwortungsbewusste Wissenschaftler als ,honest broker“ agieren sollte, und dass die Politik sich darauf besinnen sollte, nur wissenschaftlich lösbare Fragen an die Wissenschaft zu stellen, nicht aber der eigenen Verantwortung auszuweichen - in normativ schwierigen Situationen eine werte-konsistente „Lösung“ zu finden.

\subsection{Risiken der Kommunikation}

Peter Weingart und seine Kollegen haben in ihrem Buch Von der Hypothese zur Katastrophe (Weingart et al. 2002; s. a. Weingart et al. 2000) rekonstruiert, wie das Klimathema in Deutschland aus der Wissenschaft in den politisch-medialen Bereich kam.

Zunächst gab es in der Wissenschaft eine „Anthropogenisierung und Politisierung“, wonach erstens der Mensch am Klimawandel Schuld sei, und er diesen auch durch verantwortungsbewusstes Verhalten steuern und bewältigen könne. Verursacher, Betroffene 
und Handlungsoptionen wurden klar benannt. So hieß es in der 1986er Erklärung des Arbeitskreises Energie der Deutschen Physikalischen Gesellschaft: „Um die drohende Klimakatastrophe zu vermeiden, muss [...] jetzt [...] damit begonnen werden, die [...] Emission der [...] Spurengase drastisch einzuschränken“ (Arbeitskreis Energie 1986).

Diese Darstellung fand schnell Eingang in die politische Diskussion, auch weil sie sich für einen breiteren politischen Umweltdiskurs eignete. Dabei wurde der einmal in die Welt gesetzte Katastrophenbegriff in die politische Sprache übernommen. Eine nationale Klimaforschungs-Expertise wurde etabliert. Gleichzeitig wurde die „Klimakatastrophe" und der Kampf dagegen als Gegenstand politischer Regulierung verstanden und beschrieben.

Das Thema wurde von den Massenmedien aufgenommen, wobei eine weitere Dramatisierung und Zuspitzung erfolgte. Weingart et al. beschrieben hier die Elemente der „Herstellung der Ereignishaftigkeit des Klimawandels“, „Die Inszenierung der Alltagsrelevanz des Klimawandels“ und schließlich die „Transformation der wissenschaftlichen Hypothese in die Gewissheit der kommenden Katastrophe“ (Weingart et al. 2002).

Sie belegen alle diese Schritte mit Beispielen. Dann stellen sie die Frage nach den Risiken für die drei Akteure Wissenschaft, Politik und Medien.

Bei der Wissenschaft ist es vor allem der „Glaubwürdigkeitsverlust durch die Eigendynamik der Katastrophenmetapher". Dieser Begriff hat den Auftritt der Klimaforschung auf der politisch-medialen Bühne ermöglicht, aber er hat mit seinem kulturellen Konstrukt auch eine Reihe von Konnotationen mit eingeschleust. Mit diesen wird die Wissenschaft sodann wieder konfrontiert: Ihr habt dies und jenes behauptet, wie passt dies mit dieser und jener aktuellen Entwicklung zusammen? Klaus Hasselmann analysierte dies Phänomen in seiner Replik „Die Launen der Medien“ (Hasselmann 1997; s. a. http://www. rz.shuttle.de/rn/sae/warming/klima972.htm) und beklagte, dass die wissenschaftlichen Aussagen zunächst eine Metamorphose durchmachen würden, und dass die Wissenschaft sich an diesen mutierten Aussagen messen lassen müsse. Das ist sicher nicht fair, aber politisch-soziale Realität. Oder wie ein Journalist mir einmal sagte: „,Wer mit den Medien nach oben fährt, fährt mit ihnen auch wieder herunter". In beiden Fällen funktionieren die Fahrstühle nach den gleichen Regeln.

Für die Politik besteht das Risiko in der Möglichkeit, dass die so gesteckten Ziele nicht erreicht werden. Weingart et al. sprechen von einem Legitimationsverlust durch Selbstüberforderung. Dass das Kyoto-Protokoll „die Klimakatastrophe“ nicht hat „,verhindern“ können, war von Anfang an absehbar; und die einseitige Fokussierung auf die Energiepolitik war für die öffentliche Inszenierung zwar nützlich, wurde aber den Sachfragen nicht gerecht.

Die Medien fürchten vor allem den „Verlust der Aufmerksamkeit des Publikums“, weil Konzepte und Begrifflichkeiten sich abnutzen. Wenn 2005 erklärt wird, man habe nur noch 10 Jahre zur Rettung des Klimas zur Verfügung (z. B. McCarthy 2005), ${ }^{6}$ und dann in den Folgejahren außer Rhetorik und symbolischen Akten wenig passiert, weder auf der wissenschaftlichen noch auf der politischen Seite, dann wird auf der Seite der Medien versucht, die Aufmerksamkeit des Publikums anderweitig zu erringen, z. B. durch die Propagierung eines skeptischen Gegendiskurses (z. B. Der Spiegel 19/2007:

6 BILD titelte in 2007, dass noch 13 Jahre bis zum Weltuntergang blieben. 
„Abschied vom Weltuntergang“). Genau dies ist in den letzten Jahren zu beobachten. Dieser medial inszenierte Gegendiskurs folgt der Logik der Hasselmannschen „Launen der Medien“, aber auch dem Versuch innerhalb der Naturwissenschaft, das kulturelle Konstrukt zugunsten des ,wissenschaftlichen“ Konstrukts zurückzudrängen. Weingart und seine Mitarbeiter beschreiben das so: „Gegenstand und Auslöser der [Klima-]Skepsis sind nicht zuletzt die Korrekturen und Relativierungen der wissenschaftlichen Klimaszenarien durch die etablierte Klimaforschung selbst. Was in der Wissenschaft ein normaler Vorgang ist, wird in den Medien zum Anlass von Misstrauen." (Weingart et al. 2002: 141)

Die Wissenschaft, genauer: die wissenschaftlichen Einrichtungen reagieren auf dies Risiko durch die Implementierung professioneller „Pressearbeit“ - die sich an „massenmedialen Repräsentationsprinzipien“ orientiert. Die Politik sichert sich ab durch eine Hierarchisierung von Wissen und durch Beratung, mit Kanzlerberatern, Climate Service Centern und ähnlichem. Die Massenmedien suchen die Aufmerksamkeit des Publikums durch selektive Präsentation von wissenschaftlichen Ergebnissen, die entweder im Einklang oder im Konflikt mit dem kulturellen Konstrukt stehen, oder aber durch Inszenierung von Kontroversen, womit wiederum ein kulturelles Konstrukt bedient wird, nämlich das der angeblichen Beliebigkeit wissenschaftlicher Aussagen.

\section{Maßnahmen im eigenen Hause}

Das Institut für Küstenforschung der $\mathrm{GKSS}^{7}$ sieht sich einem Beratungsbedarf vor allem aus dem Bereich des Küstenschutzes, aber auch des Seeverkehrs, des Tourismus und anderen Sektoren konfrontiert. Ebenso gewichtig ist der Deutungsbedarf durch die Öffentlichkeit, vor allem in Form der Medien, zum Klimawandel und zur Einordnung von auffälligen Ereignissen, vor allem Stürmen und Sturmfluten. In anderen Einrichtungen, sei es das Max Planck Institut für Meteorologie in Hamburg oder das Alfred Wegener Institut für Polar- und Meeresforschung in Bremerhaven, sieht es ähnlich aus. Die Institutsleiter reisen durch die Lande und erklären einem interessierten Publikum die infrage stehenden Phänomene und Reaktionsmöglichkeiten.

Diese Vortragsreisen wurden zunächst kurzfristig entsprechend dem konkreten Bedarf von Fall zu Fall organisiert. Inzwischen haben wir versucht, die Aufgabe breiter und systematischer anzugehen. Die Elemente dieses Ansatzes sind:

1. Eine systematische Überlegung über die Bedeutung von Anpassung an den Klimawandel und Möglichkeiten der Verminderung des Klimawandels, etwa in Form des 10-punktigen „Zeppelin Manifests“ von Nico Stehr und mir selbst (vgl. Stehr u. von Storch 2008).

2. Eine Zusammenstellung der wissenschaftlich legitimierten Wissensansprüche zum gegenwärtigen und zukünftigen Klimawandel in relevanten Regionen. „Wissenschaft-

7 Das GKSS-Forschungszentrum Geesthacht ist eine der Einrichtungen der Helmholtz Gemeinschaft Deutscher Forschungszentren. GKSS ist der Name der Einrichtung, keine Abkürzung. Siehe auch http://www.gkss.de. 
lich legitimiert“" steht hier praktisch für Publikationen aus anerkannten wissenschaftlichen Einrichtungen, die der gängigen guten wissenschaftlichen Praxis entsprechen. Es geht hier um die Darstellung nicht des „,besten Wissens“, was ohnehin häufig ein problematischer Anspruch ist, sondern um die Bestimmung des Konsensus, einschließlich den konsensualen Feststellungen dessen, worüber es eben keinen Konsens gibt. Konsens des Dissenses (vgl. The BACC author team 2008).

3. Die Verfügbarkeit von raum-zeitlich detaillierten Beschreibungen der klimatischen regionalen Veränderungen in der jüngeren Vergangenheit. Für Nordeuropa ist ein derartiger Datensatz CoastDat (vgl. http://www.coastdat.de/), der den Zeitraum seit 1948 bis heute abdeckt. Neben anderen vernünftigen Anwendungen (vgl. Weisse et al. 2009) erlaubt so ein Datensatz die Einschätzung, inwieweit derzeitige Veränderungen als konsistent mit jenen Änderungen angesehen werden dürfen, die uns Klimamodelle in Form von Szenarien angeben (vgl. Bhend u. von Storch 2009). In der Kommunikation mit Medien, Politik und Entscheidungsträgern wird ja gerne und oft schlampig davon geredet, dass dies und jenes Ereignis ein Vorbote dessen sei, was da auf uns zukommen wird. Konkret belegt wird dies in der Regel nicht, was zumindest im Falle der fachnahen Beratung zur guten wissenschaftlichen Praxis gehören sollte.

4. Abgerundet wird dieser Katalog an Wissensangeboten durch einen Wissensmakler, ein regionales Klimabüro. Im Rahmen der Helmholtz Gemeinschaft haben wir vier derartige regionale Klimabüros eingerichtet (vgl. http://www.klimabuero.de): Das Klimabüro der GKSS kümmert sich um den Bereich Norddeutschland, speziell auch um Aspekte der Küste, also Stürme, Sturmfluten, Seegang; das Karlsruher Büro nimmt sich des Südens an, das Leipziger Büro der Mitte und das Bremerhavener der arktischen Regionen. Das Ganze arbeitet im Verbund mit verwandten Aktivitäten des Deutschen Wetterdienstes und des Climate Service Centers ab 1. Januar 2009. Der Grundgedanke der regionalen Klimabüros beruht auf der Beobachtung, dass die Klimakommunikation keine Frage von „Knowledge speaks to power“ oder der Nachhilfe einer ungebildeten Öffentlichkeit sei, wie naive Physiker und Meteorologen immer noch gerne glauben. Es geht nicht um eine pädagogisch wertvolle Webseite, ein Klimaspiel. Vielmehr besteht auf Seiten der Wissenschaft die Notwendigkeit zunächst zu verstehen, worin denn die Fragen von Öffentlichkeit und Politik überhaupt bestehen, wie diese Fragen mit anderen Komplexen verknüpft sind, ob die Antworten der Klimaforschung überhaupt einen Bezug zu diesen Fragen haben, und inwieweit unsere naturwissenschaftlichen Konzepte in Konkurrenz zu kulturellen Konstrukten stehen.

Nach diesem Ausflug in eine eher operationelle Dimension, wie Klimaforschung sich angemessen im öffentlich-politischen Raum darstellen kann, beende ich meinen Rundblick mit einer zusammenfassenden persönlichen Schlussbemerkung.

\section{Schlussbemerkung}

Naturwissenschaftliches Wissen ist in der gesellschaftlichen Praxis nur eine Form des Wissens; es muss mit anderen Formen konkurrieren, und gewinnt diese Konkurrenz nicht automatisch. Ohne Kenntnis dieser Dynamik wird die Klimaforschung versuchen, die 
Verbreitung der eigenen Botschaft durch propagandistische Tricks wie Zuspitzung oder zweckorientierte Selektion zu „optimieren“. Dadurch wird die Öffentlichkeit entmündigt und die Wissenschaft als sozial akzeptierte Einrichtung beschädigt. Ich empfinde es als unsere Aufgabe, Wissenschaft nachhaltig zu betreiben. Und als Teil der Öffentlichkeit möchte ich persönlich nicht entmündigt werden.

\section{Literatur}

Arbeitskreis Energie (AKE) der Deutschen Physikalischen Gesellschaft. 1986. Warnung vor der drohenden Klimakatastrophe. Frankfurter Rundschau, 19.9.

BACC author team. 2008. Assessment of climate change in the Baltic Sea Basin. Berlin: Springer Verlag.

Bhend, Jonas, and Hans von Storch. 2009. Is greenhouse gas forcing a plausible explanation for the observed warming in the Baltic Sea catchment area? Boreal Environment Research 14: $81-88$.

Brikowski, Tom H., Yair Lotan, and Margarete S. Pearle. 2008. Climate-related increase in the prevalence of urolithiasis in the United States. Proceedings of the National Acadamy of Science 105: 9841-9846.

Fleck, Ludvik. 1980. Entstehung und Entwicklung einer wissenschaftlichen Tatsache: Einführung in die Lehre vom Denkstil und Denkkollektiv. Frankfurt a.M.: Suhrkamp.

Funtowicz, Silvio O., and Jerome R. Ravetz. 1985. Three types of risk assessment: a methodological analysis. In Risk Analysis in the Private Sector, Hrsg. C. Whipple and V.T. Covello, 217-231. New York: Plenum.

Hasselmann, Klaus. 1997. Die Launen der Medien. Die Zeit 31, 25.07.

Lippitt, Ronald. 1968. Kurt Lewin. In International Encyclopedia of the Social Sciences, Hrsg. David Sills, 266-271. Bd. 9. New York: Macmillan \& Free Press.

McCarthy, Michael. 2005. Countdown to global catastrophe. The Independent online edition, January 24 .

Milne, Anthony. 1989. Our drowning world. London: Prism Press.

Pielke, Roger A. jr. 2007. Mistreatment of the economic impacts of extreme events in the Stern Review Report on the economics of climate change. Global Environmental Change 17: 302-310.

Pielke, Roger A. jr. 2007.The honest broker. Cambridge: Cambridge University Press.

Stehr, Nico, und Hans von Storch. 1999a. Wetter, Klima, Mensch. München: Beck Verlag.

Stehr, Nico, und Hans von Storch. 1999b. An anatomy of climate determinism. In Wissenschaftlicher Rassismus - Analysen einer Kontinuität in den Human- und Naturwissenschaften, Hrsg. Heidrun Kaupen-Haas und Christian Saller, 137-185, Frankfurt a.M.: Campus.

Stehr, Nico, und Hans von Storch. 2008. 10-Punkte Manifest: So kann Deutschland den Klimawandel bewältigen - spiegel online. http://www.spiegel.de/wissenschaft/natur/0,1518,57603211,00.html.

Weingart, Peter, Anita Engels, and Petra Pansegrau. 2000. Risks of communication: Discourses on climate change in science, politics and the mass media. Public Understanding of Science 9: 261-283.

Weingart, Peter, Anita Engels, und Petra Pansegrau. 2002. Von der Hypothese zur Katastrophe. Opladen: Leske + Budrich. 
Weisse, Ralf, Hans von Storch, Ulrich Callies, Alena Chrastansky, Frauke Feser, Iris Grabemann, Heinz Günther, Andreas Plüß, Thomas Stoye, Jan Tellkamp, Jörg Winterfeldt, and Katja Woth. 2009. Regional meteo-marine reanalyses and climate change projections: Results for Northern Europe and potentials for coastal and offshore applications. Bulletin of the American Meteorological Society (im Druck).

Yohe, Gary W., and Richard S.J. Tol. 2008. The Stern review and the economics of climate change: An editorial essay. Climatic Change 89: 231-240. 